\title{
2 On the Nature of Irrationality
}

\section{Edgar Cambaza}

\author{
Laboratory of Food Process Engineering, Graduate School of Agricultural Science, Hokkaido University, \\ Sapporo, Hokkaido, 060-0808 Japan \\ Department of Biological Sciences, Faculty of Sciences, Eduardo Mondlane University, Av. Julius Nyerere, nr. \\ 3453 Maputo, Mozambique \\ * Correspondence: edy@bpe.agr.hokudai.ac.jp; Tel.: +81-80-2876-1106
}

\begin{abstract}
It has been a challenge to visualize in nature some concepts from abstract subjects such as mathematics and philosophy. Irrationality is certainly among the least comprehensive. This essay aimed to propose that irrationality is simply an illusion resulting from the human inability to grasp the full nature of reality, rather than actual continuity flaws in the fabric of existence. There are three major arguments: (1) human senses have limitations and these are likely to be extended to the intellect, (2) there are no observable existential discontinuities in everyday experience, and (3) some rational phenomena have irrational components. These arguments point towards the existence of a physical existence of irrational quantities or distances, visible to the human eye but not fully comprehended through arithmetic abstraction.
\end{abstract}

Keywords: Irrationality, human senses, human mind, material continuity, existence.

\section{Introduction}

The notion of quantity is important for comparisons and decision-making, and numbers have been using as abstract tools to represent amounts, relationships or orders of objects across civilizations since the dawn of civilization [1]. The role of numbers was decisive for the development of virtually any field of activity requiring quantification or organization of items, from engineering to business, education, computer science, communication and many other fields. Natural numbers are perhaps the most comprehensive because it is easy to associate them to very intuitive operations involving everyday objects. This is the certainly reason why common curricula of mathematics first introduce natural numbers to students and later the contents become more complex.

It is challenging to introduce the concept and properties of negative and irrational numbers, particularly when it comes to preventing the prior knowledge of natural numbers from undermining the comprehension about the irrational [2,3]. Indeed, this problem seems to transcend mathematics if the numbers are meant to represent the objective reality. The concept of irrationality requires a level of abstraction that defies the everyday experience of the common citizen. For instance, the human mind cannot visualize $\pi$ oranges or e American dollars, although it is possible to estimate these values.

What if the problem is essentially biological? What if irrationality is just an illusion created by the human mind rather than a property inherent to nature? In other words, what if irrational quantity is part of the objective reality but the human mind lacks the intellectual framework to perceive it? If reason is taken as a component of the cognitive system [4], it should be reasonable to admit that it presents flaws just like any other. It would perhaps be naïve to assume that a common human being has the ability to fully perceive the nature of reality.

This is a brief discussion on what irrationality represents, why it is not completely determined by the human mind as a finite element, and most importantly, why it might simply be an intellectual mechanism to ensure harmony between humans and their niche in the fabric of reality. 


\section{Proposition}

This essay proposes that irrationality is a human inherited illusion resulting from the ability to survive with no need to completely understand the nature of reality. This thesis rested upon three major arguments: human limitations, existential continuity and the relationship between the rational and irrational. These arguments are inter-related as some examples can illustrate more than one argument. Yet, it is important to discuss each argument separately for the sake of clarity and objectivity.

Before going any further, it is important to keep two things in mind:

- This essay is focused on the concept of irrationality according to the set theory of mathematics, using irrational numbers as examples, to facilitate the discussion and prevent any misunderstanding. These numbers apparently cannot be obtained by dividing two integers because they result in infinite quantity of digits when represented in positional numeral systems [5]. Thus, the current definition of the term "irrationality" is not synonymous with the inability to make wise decisions, ignorance or "dumbness";

- Irrationality shall not be confused with some concepts currently poorly understood just because of their complexity. For instance, millennium challenges are not really irrational because it is believed that they will eventually be solved. As far as mainstream science indicates, numbers like $\pi$ or e present infinite decimal places and it would be impossible or even unnecessary to determine their exact value. Boldly stating, they are rationally inconceivable by the human mind and they are unlikely to ever be fully determined if intelligence never goes beyond the current state.

\section{Arguments}

\subsection{Human limitations}

If several animals do not exhibit the ability to solve certain logical operations apparently simple for humans, we might as well be unable to attain certain levels of intelligence. Irrationality has been identified numerous times but never completely deciphered, possibly because of the way the human mind works. Human senses are known to present limitations. For instance, eagles have better visual acuity, dogs have a more capable olfactory system and bats have the ability to emit and detect some sounds unintelligible for the human audition. These animals have abilities literally superhuman, capable of obtaining more precise and accurate information of certain components of reality in relation to hominids. This fact would be enough to consider that humans might not have the full notion of the surrounding reality, but paradoxes further reduce the human potential to integrally understand the existential matrix. For example, the concept of infinity has been associated with several paradoxes throughout history [6] because the experience shows plausibility for both finitude and infinitude. On one hand, the experience shows that everything has a limit but on the other hand the intuition consistently asks what comes after the end. Such incapacity to understand complex concepts like infinity or irrational numbers, combined with the limitation of human senses, certainly castrates humanity from the aptitude to truly understand how reality is.

But it is important to understand that limited intellect does not imply inability to survive. It does not seem entirely necessary to understand the irrational to be able to comprehend its effect in the surrounding reality and use it for our benefit, the same way we cannot touch a flame but it is essential since the dawn of civilization. There are also several plants with healing effects from which the active compounds have still not been isolated. Sometimes the priority is to "embrace" the mystery rather than unravel it. Indeed, the intellect's incapacity to completely understand the irrational may increase the probability of survival by limiting the acquisition of irrelevant information. Otherwise, humans and many other living beings would be unable to thrive as far as they did. From a Darwinian point of view, human attributes can be a combination of characteristics assuring its niche [7], and this logic seems applicable to all range of agents making up the existential matrix. From bacteria to plants and animals, all living beings seem adapted to their specific 
environments and such adaptation is certainly not restricted to physical features. The instincts and even intelligence are perhaps also optimized to their respective niches.

What if we tend to have just the minimum requirements for survival? It would make sense if this time we consider a more Lamarckian perspective. According to Jean Baptiste Lamarck, a certain trait becomes prevalent among a species as the organisms use it more frequently [8], and one should expect as result that the dominant traits of a species is the one it has mastered over time. Unnecessary or obsolete traits end up disappearing or never existing in the first place. Regardless if one explores a Darwinian or Lamarckian perspective, it is reasonable to presume that the current average level of intelligence among humans is what humanity needed so far for survival or, in general, for people's necessities, basic or not.

Recent studies suggest that the overall intelligence quotient (IQ) has been increasing considerably (the Flynn effect) $[9,10]$, possibly due to the development of science and technology, and consequent exposure to increasingly demanding intellectual challenges. This can explain the high awareness of the contemporary intellectual limitations including the concept of irrational components in the fabric of reality. Moreover, this increase in intelligence is perhaps the reason why old mathematical challenges such as Catalan's, Kepler, Poincaré conjectures have been solved recently in considerably short time span [11-13], though in some cases the development of powerful tools such as computation might have played a role. Thus, the limited perception of the irrational does not seem to be a limiting factor for human survival but the current application of concepts such as $\pi$ and e in science and engineering fields shows how important they are and they might become understood in more depth or even in plenitude in the future.

In summary, the argument of human limitations suggests that the intellect present limitations just like any other physical feature including the senses. The mind is "blind" for irrationality the same way the eyes and ears cannot capture some frequencies, and the nose cannot smell some chemicals. It also takes in consideration the fact that all animals seem to exhibit limited intellect. As animal, the human species is not likely to be an exception.

\subsection{Existential continuity}

From a human perspective, irrationality is like a continuity anomaly in the otherwise coherent logical reasoning. It can be compared to a vault with hidden lock: it can be located, its surroundings can be understood but its content remains a mystery. The reason why it exists is also unknown and it just demonstrates how limited the human mind is. Irrationality's undefined nature is perhaps why it is object of speculation, where frequently people tend to believe is located the "fire from which comes the smoke of existence". This tendency to associate all superstitions to the irrational as part of a "great common truth" is quite similar to the way individuals use a trash bin: any unsettling thing is dropped there to clean up the environment and it provides certain comfort, at least temporarily. But this mentality resembles the anecdote about two men who believed to be namesakes because they could not remember their names. For instance, $\pi$ and $e$ are both irrational but they are not the same number as far as the current understanding shows.

It is important to notice that irrationality does not seem to compromise the integrity of existence or human affairs, since it is possible to live aware or not of it. It might mean that irrationality results from human limitations, rather than flaws in the absolute reality. Otherwise, would it not result in some form material instability? In other words, irrational quantities and relations are perhaps existing phenomena in nature but the human mind is simply "blind" for them, the same way eyes or ears cannot perceive certain electromagnetic waves. For instance, if we fill up a $4 \mathrm{~L}$ jar at some point we have to cross $\pi \mathrm{L}$ (approximately 3.14 L). The water does not seem to skip $\pi \mathrm{L}$ and our vision will not show a gap at that point, $e \mathrm{~L}, \sqrt{2} \mathrm{~L}$ or any other of the several infinite decimals between $0 \mathrm{~L}$ and $4 \mathrm{~L}$ (at least intuitively or according to the observable experience). Furthermore, if the jar is full the intuition does not suggest that the quantity of water is any less than $4 \mathrm{~L}$. It appears acceptable to conclude that the full quantity of water is visible, including the irrational component of the volume. One could argue that the particular irrational values are reflected in quantities so small that the naked eye is incapable of detecting, but if the quantities form a continuum of infinite values, the 
combined quantity of irrational values would be impossible to ignore. Indeed, it would be a Zeno's paradox [14] and theoretically we could never fill up the jar.

The problem of irrational quantities seems intellectual rather than physical. Only the idea of the location of a particular irrational volume is not fully intelligible for the human mind. Anyway, no tool currently available would be capable of setting or measuring such kind of quantity if we assume that material world is composed by particles and the irrational quantities have infinitely decimal nature. In this case, irrationality is an intellectual illusion, or otherwise the paradox would be even more complex. For instance, assuming that every point in space is at a $\pi$ distance of several other points around yet the human mind has the ability to perceive it, it means that only the concept of $\pi$, not the material world or the $\pi$ distance itself, is unintelligible. Furthermore, given $r$ as the radius of a circumference, it would be impossible to visualize its area $\left(\pi r^{2}\right)$ or perimeter $(2 \pi r)$ because both are products of $\pi$, but circles are among the most recognizable shapes in nature.

If the concepts of geometric irrationality were projected to the objective world, there would probably be more frequent noticeable continuity errors or incoherent forms. Instead, reality is so coherent that several literati support the theory of intelligent design [15]. Indeed, the infinite nature of the irrational might be the glue assuring the continuity between the discreet units of rationality. Thus, irrationality seems to be mostly an idea "haunting" logical objects or operations, with no perceptible representation in the objective reality. Perhaps human senses (vision, audition, olfaction) or their cognitive paths are set to somehow mask such incoherencies as a defense mechanism to ensure the mental integrity and ultimately our survival. In other words, our inability to comprehend the irrational might be a mental adaptation to the environment. From this perspective, every animal would have its own profile of rationality set to work according to its niche and the true shape of nature might even not be fully understood by any creature.

In summary, this argument states that irrationality is an illusion because several particles mathematically classified as irrationally positioned in relation to some reference position or quantity are still clearly detectable as parts of the observable reality. There are no continuity lapses in reality due to irrational quantities and positions. Maybe the senses or even the intellect masks such gaps to allow them to thrive in an otherwise nonsensical world.

\subsection{Irrationality within the rational world}

It is perhaps impossible to know how far the irrational affects the nature of reality because it is incomprehensible by definition. The value of irrationality can only be perceived through its occurrence in nature's important components or phenomena, sometimes in very elegant concepts such as the proportion between the perimeter and diameter of a circumference, or its participation in highly organized patterns such as spirals and trigonometric relations. Trigonometry is a cornerstone to understanding oscillations or waves and its application ranges from music and seismology to cardiology or any other discipline recurring to functions with repetitive or periodical dependent variables. One can state that nature is like a very well crafted fabric with hidden finish.

How can irrationality be part of very simple shapes such as circles, spirals, waves, the hypotenuse of a triangle rectangle with 1 as cathetus, or even the pattern at which microbes grow (calculated through natural logarithms) [16,17]? Considering the chaos theory [18], should not irrational elements originate even less comprehensive elements? The latter question certainly has flaws (e.g. non-visible cells form well-known organisms) but it is worth wondering why simple shapes result from such obscure concepts. But the lack of knowledge on what the irrational really is opens up the possibility that they might be either too complex or very simple, and if the latter is true perhaps the currently known combination between irrational quantities or proportions among them or with rational ones do not originate enough chaos to result in incomprehensible phenomena. Or perhaps the rational component of nature, not being infinitely small, ends up dominating the matrix of reality the same way an extremely diluted solution of sugar barely shows any sweetness. In any case, irrationality is present in rational shapes and elements as an integrant part of nature.

The mysterious nature of the irrational opens space to speculation. For instance, which other aspects of reality and how much it affects? Despite of the "objective" irrational determined through 
the logical subjects developed so far such as arithmetic or algebra, are not there more irrational elements as relevant for existence as we know it? And even the already known forms of irrational, how can humanity some day realize the full impact on reality if our mind is unable to copiously understand them? Furthermore, what can result in reality from their combination? And if the irrational is compared to a tunnel in which we enter but never find the end, which "ghosts" are hidden in its "darkness"? There is no shortage of speculations around these questions but it seems reasonable to assume that whichever answers come to surface, nature is built up with participation of truly irrational proportions, measures and quantities.

In summary, there are irrational components of the rational reality and their concepts seem inseparable (e.g. perimeter of a circumference requires $\pi$ to be calculated). Thus, irrational elements have exact "size" in nature or even in the abstract realm. Thus, reality does accommodate the irrational and the human mind is the factor preventing us to accurately visualize the true nature of irrationality.

\section{Conclusion}

The current discussion suggests that irrationality is an intrinsic human trait, resulting from the inability to fully grasp reality. As far as it seems, irrationality is not a constraint to everyday life of the common men, operating as a hidden component of the objective reality. Irrationality appears to be more relevant for academics, engineers, philosophers and other professionals dealing with abstract subjects. It is possible to imagine that it was possible to live a healthy and "fulfilled" life even when virtually the entire humanity was not aware of the irrational, at least at the current level of abstraction. Moreover, several other living beings with no record of the human level of intellect have shown the ability to perpetuate their existence.

Assuming that human limitations are likely to be not only physical but also intellectual, the material world does not show existential intervals of continuity even though logic proposes their existence, and irrational proportions, quantities, distances and sizes are integral part of the rationally known reality, it is reasonable to assume that irrationality is a human illusion misrepresenting the totality of the surrounding existence.

Funding: This research received no external funding.

Conflicts of Interest: The author declares no conflict of interest.

\section{References}

1. Corry, L. A brief history of numbers. First edition. ed.; Oxford University Press: Oxford, United Kingdom ; New York, NY, United States of America, 2015; p xiii, 309 pages.

2. Van Hoof, J.; Verschaffel, L.; Van Dooren, W. Inappropriately applying natural number properties in rational number tasks: Characterizing the development of the natural number bias through primary and secondary education. Educational Studies in Mathematics 2015, 90, 39-56.

3. Van Dooren, W.; Lehtinen, E.; Verschaffel, L. Unraveling the gap between natural and rational numbers. Learning and Instruction 2015, 37, 1-4.

4. Sternberg, R.J.; Sternberg, K. Cognitive psychology. Sixth Edition ed.; Wadsworth: Belmont, California, United States of America, 2009.

5. Boyer, C.B.; Pérez, M.M. História da matemática. 3ª Edição. São Paulo: Blucher 2012.

6. Dubinsky, E.; Weller, K.; Mcdonald, M.A.; Brown, A. Some historical issues and paradoxes regarding the concept of infinity: An apos-based analysis: Part 1. Educational Studies in Mathematics 2005, 58, 335-359.

7. Orr, H.A. The genetic theory of adaptation: A brief history. Nature Reviews Genetics 2005, 6, 119.

8. Gissis, S.; Gissis, S.B.; Jablonka, E.; Zeligowski, A. Transformations of lamarckism: From subtle fluids to molecular biology. MIT press: 2011. 
243 9. Baker, D.P.; Eslinger, P.J.; Benavides, M.; Peters, E.; Dieckmann, N.F.; Leon, J. The cognitive impact of the 244 education revolution: A possible cause of the flynn effect on population iq. Intelligence 2015, 49, $144-158$.

245 10. Flynn, J.R. Requiem for nutrition as the cause of iq gains: Raven's gains in britain 1938-2008. Economics \& $246 \quad$ Human Biology 2009, 7, 18-27.

247 11. Hales, T.C. A computer verification of the kepler conjecture. arXiv preprint math/0305012 2003.

248 12. Milnor, J. Towards the poincaré conjecture and the classification of 3-manifolds. Notices AMS 2003, 50, 1226-1233.

250 13. Metsänkylä, T. Catalan's conjecture: Another old diophantine problem solved. Bulletin of the American 251 Mathematical Society 2004, 41, 43-57.

252 14. Albers, S.A. An attitude of complexity: Thirteen essays on the nature and construction of reality under the

254 15. Shanks, N. God, the devil, and darwin: A critique of intelligent design theory. Oxford University Press: 2004.

255 16. Zwietering, M.; Jongenburger, I.; Rombouts, F.; Van't Riet, K. Modeling of the bacterial growth curve. Applied 256 and environmental microbiology 1990, 56, 1875-1881.

257 17. Buchanan, R.L.; Whiting, R.C.; Damert, W.C. When is simple good enough: A comparison of the gompertz, 258 baranyi, and three-phase linear models for fitting bacterial growth curves. Food microbiology 1997, 14, 313-326.

259 18. Thietart, R.-A.; Forgues, B. Chaos theory and organization. Organization science 1995, 6, 19-31. 